\title{
PHASE SPECTRA SEEN FROM SPACE
}

\author{
TH. STRAUS \\ Osservatorio Astronomico di Capodimonte, Napoli, Italy \\ F.-L. DEUBNER \\ Institut für Astronomie und Astrophysik, Würzburg, Germany \\ B. FLECK \\ ESA Space Science Department, NASA/GSFC, Greenbelt, USA \\ C. MARMOLINO \\ Università "Federico II", Napoli, Italy \\ G. SEVERINO \\ Osservatorio Astronomico di Capodimonte, Napoli, Italy \\ AND
T. TARBELL
Lockheed-Martin Advanced Technology Center, Palo Alto, USA
}

A 15 hour time series of simultaneous spectroscopic measurements of the continuum intensity, Doppler shift, and line depth in the Ni I $6768 \AA$ line at disk center of the quiet sun has been obtained during a guest investigator campaign with the Michelson Doppler Imager (MDI, see Scherrer et al. 1995). In addition, the line intensity has been calculated from the continuum intensity and the line depth data. The spatial shifts due to solar rotation have been approximately removed during the observation run by shifting the CCD read-out area by 1 pixel every 4 minutes. Small residual shifts have been removed during the data analysis. Before applying the Fourier analysis, a (temporal) low pass filtered mean velocity (line of sight component of solar rotation) and mean intensity (center-limb variation) has been removed from each frame. Finally, the continuum intensity $(C)$, line intensity $(I)$, and velocity $(V)$ time series have been subjected to a 3D Fourier transform, yielding $k-\omega$ power, cross power, phase difference, and coherence spectra. The data can now be compared to recent ground-based observations (Deubner, 1990; Deubner et al., 1992; Straus \& Bonaccini,1997).

The data from space conclusively prove the existence of different phase regimes in the $k-\omega$ diagram (see Fig. 1), as indicated from ground-based observations. These different regimes, in part theoretically understood (see Fig. 2-6 of Beckers 1981 for a summary of phenomena in the $k-\omega$ diagram), in part to be investigated in more detail, are:

(1) the p-modes showing negative $V-I$ phase values and an upward progressive component; they can now be followed up to $8.3 \mathrm{mHz}$ and even to higher frequencies 

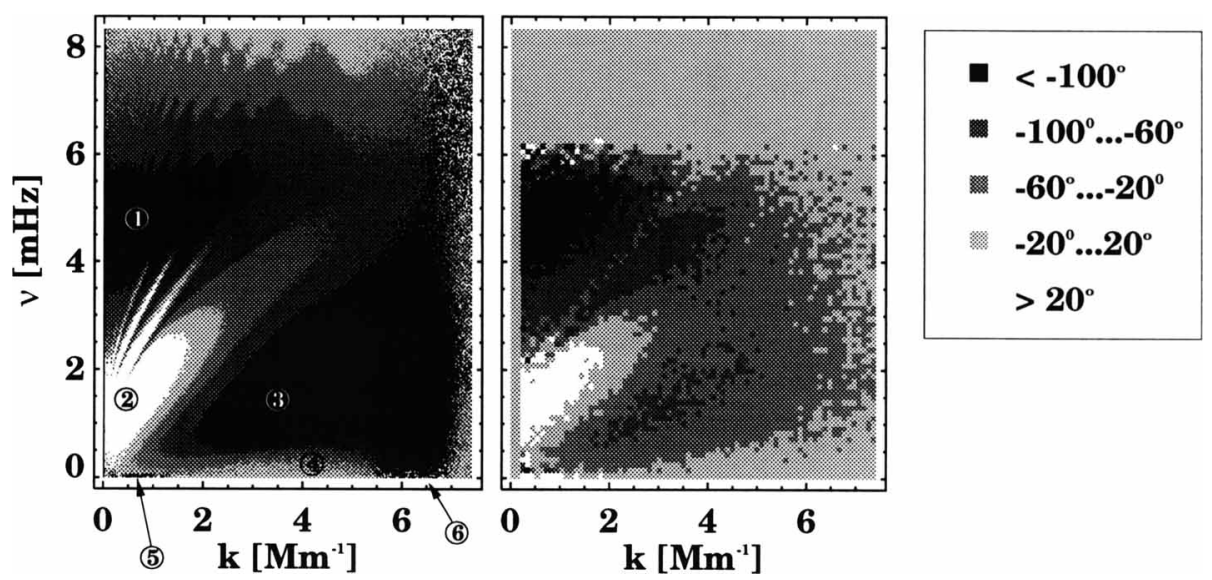

Figure 1. Comparison between $k-\omega$ phase difference spectra between the velocity and the intensity fluctuations, as observed from space (left panel) and from ground (right panel, Straus \& Bonaccini, 1997); the labels refer to the text.

(see Jefferies, this conference, for a review on high frequency pseudo modes). (2) a low power, intermediate coherence plateau showing positive $V-I$ phase, which continues to higher frequencies into the space between the ridges. This regime has been attributed to downward propagating waves (Marmolino et al., 1993), which can now clearly be demonstrated by the $I-C$ phase spectrum. (3) A distinct regime of negative $V-I$ phase, which has been attributed to internal gravity waves in the atmosphere (Deubner et al., 1992), as indicated by the downward propagating character. (4) A nearly zero $V-I$ phase regime corresponding to the convective overshoot; this regime is limited to spatial wavenumbers less than $5.5 \mathrm{Mm}^{-1}$. Furthermore, two other regimes can be identified which merit further investigations: (5) a regime at very long periods and spatial wavenumbers up to $1.5 \mathrm{Mm}^{-1}$, which shows anticorrelation between $V$ and $I$, recently surmised as being due to the chromospheric network (Straus \& Bonaccini, 1997); and (6) for the first time visible, a regime at very high spatial wavenumbers which also shows a clear anticorrelation between $V$ and $I$. Fine scale magnetic field structures might be a candidate for the latter phenomenon.

\section{Acknowledgements}

This work has been partially funded by ASI contract ASI 95-RS-69.

\section{References}

Beckers, J. M.: 1981, in Jordan, S. (ed.), The Sun as a Star, NASA SP 450.

Deubner, F.-L.: 1990, in Stenflo, J. O. (ed.),Solar Photosphere: Structure, Convection, and Magnetic Fields, IAU Symp. 138, p. 217.

Deubner, F.-L., Fleck, B., Schmitz, F., Straus, Th.: 1992, Astron. Astrophys. 266, 560.

Jefferies, S.: 1997, this proceedings.

Marmolino, C., Severino, G., Deubner, F.-L., Fleck, B.: 1993, Astron. Astrophys. 278, 617.

Scherrer, P.H., Bogart, R.I., Bush, J.T., et al.: 1995, Solar Phys. 162, 129.

Straus, Th., Bonaccini, D.: 1997, Astron. Astrophys 324, 704. 